\title{
Triplet-blockaded Josephson supercurrent in double quantum dots
}

\author{
Daniël Bouman $\odot,{ }^{1}$ Ruben J. J. van Gulik, ${ }^{1}$ Gorm Steffensen, ${ }^{2}$ Dávid Pataki $\odot,{ }^{3}$ Péter Boross, ${ }^{4}$ Peter Krogstrup,${ }^{2}$ \\ Jesper Nygård $\odot,{ }^{2}$ Jens Paaske, ${ }^{2}$ András Pályi, ${ }^{3}$ and Attila Geresdi $\odot^{1,5, *}$ \\ ${ }^{1}$ QuTech and Kavli Institute of Nanoscience, Delft University of Technology, NL-2600 GA Delft, The Netherlands \\ ${ }^{2}$ Center for Quantum Devices, Niels Bohr Institute, University of Copenhagen, DK-2100 Copenhagen, Denmark \\ ${ }^{3}$ Department of Theoretical Physics and MTA-BME Exotic Quantum Phases Research Group, Budapest University of Technology \\ and Economics, $H$-1111 Budapest, Hungary \\ ${ }^{4}$ Institute for Solid State Physics and Optics, Wigner Research Centre for Physics, P.O. Box 49, H-1525 Budapest, Hungary \\ ${ }^{5}$ Quantum Device Physics Laboratory, Department of Microtechnology and Nanoscience, Chalmers University of Technology, \\ SE-41296 Gothenburg, Sweden
}

(Received 12 August 2020; accepted 2 December 2020; published 21 December 2020)

\begin{abstract}
Serial double quantum dots created in semiconductor nanostructures provide a versatile platform for investigating two-electron spin quantum states, which can be tuned by electrostatic gating and an external magnetic field. In this Rapid Communication, we directly measure the supercurrent reversal between adjacent charge states of an InAs nanowire double quantum dot with superconducting leads, in good agreement with theoretical models. In the even charge parity sector, we observe a supercurrent blockade with increasing magnetic field, corresponding to the spin singlet to triplet transition. Our results demonstrate a direct spin to supercurrent conversion, the superconducting equivalent of the Pauli spin blockade. This effect can be exploited in hybrid quantum architectures coupling the quantum states of spin systems and superconducting circuits.
\end{abstract}

DOI: 10.1103/PhysRevB.102.220505

Semiconductor quantum dots, where the orbital and spin states of single localized electrons can be controlled [1], are one of the leading platforms for quantum information processing [2]. Specifically, double quantum dots (DQDs) connected in a series [3] became the preferred physical implementation of spin [4], and spin-orbit quantum bits [5] in low-dimensional semiconductor nanodevices, such as heterostructures hosting a two-dimensional electron gas or semiconductor nanowires. In these devices, the readout of the spin quantum state relies on spin-dependent single electron tunneling processes, which then enable charge readout via direct electronic transport [1], charge sensing techniques [6], or dipole coupling to a microwave resonator $[7,8]$.

In a superconducting nanodevice, the dissipationless supercurrent $I_{\mathrm{S}}$ at zero voltage bias is driven by the quantum mechanical phase difference $\varphi$ up to a maximum amplitude, $I_{\mathrm{C}}$, the critical current [9]. In the lowest order of tunneling, the supercurrent-phase relationship (CPR) [10] is sinusoidal, $I_{\mathrm{S}}(\varphi)=I_{\mathrm{C}} \sin (\varphi)$, which describes the coherent transfer of single Cooper pairs through the weak link. When the weak link is a nonmagnetic tunnel barrier, a zero phase difference is energetically favorable in the absence of supercurrent, which

\footnotetext{
*Corresponding author: geresdi@ chalmers.se
}

Published by the American Physical Society under the terms of the Creative Commons Attribution 4.0 International license. Further distribution of this work must maintain attribution to the author(s) and the published article's title, journal citation, and DOI. Funded by Bibsam. is described by a positive critical current, $I_{\mathrm{C}}>0$. In contrast, a negative coupling yields a supercurrent reversal, $I_{\mathrm{C}}<0$, often denoted a $\pi$ junction due the $\pi$ phase shift in the CPR. This negative coupling has been observed in ferromagnetic weak links [11,12], out-of-equilibrium electron systems [13], and semiconductor quantum dot junctions $[14,15]$.

The dependence of the critical current on the spin state and charge state of a DQD has also been addressed theoretically [16-22], and the recent progress in materials science of superconductor-semiconductor hybrid nanostructures [23] enabled measurements of the amplitude of the critical current as well [24,25], in correlation with the charge states of the DQD.

In this Rapid Communication, we report on direct measurements of the CPR through a DQD weak link formed by an electrostatically gated InAs nanowire. By employing a phasesensitive measurement scheme, where the DQD is embedded in a superconducting quantum interference device (SQUID), we characterize the full CPR of the DQD, enabling a signful measurement of $I_{\mathrm{C}}$. The direct observation of the supercurrent reversal in the total charge number boundaries allowed us to identify the even and odd occupied states. Finally, the magnetic field dependence of the supercurrent amplitude in the even occupied state reveals the presence of a supercurrent blockade in the spin-triplet ground state, in agreement with numerical calculations.

We built our device (Fig. 1) from an approximately $7-\mu \mathrm{m}$ long InAs nanowire grown by molecular beam epitaxy, and in situ partially covered by a 6-nm-thick epitaxial aluminum shell with a typical superconducting gap of $\Delta \approx 200 \mu \mathrm{eV}$ $[23,26]$. We formed two segments with the aluminum layer 

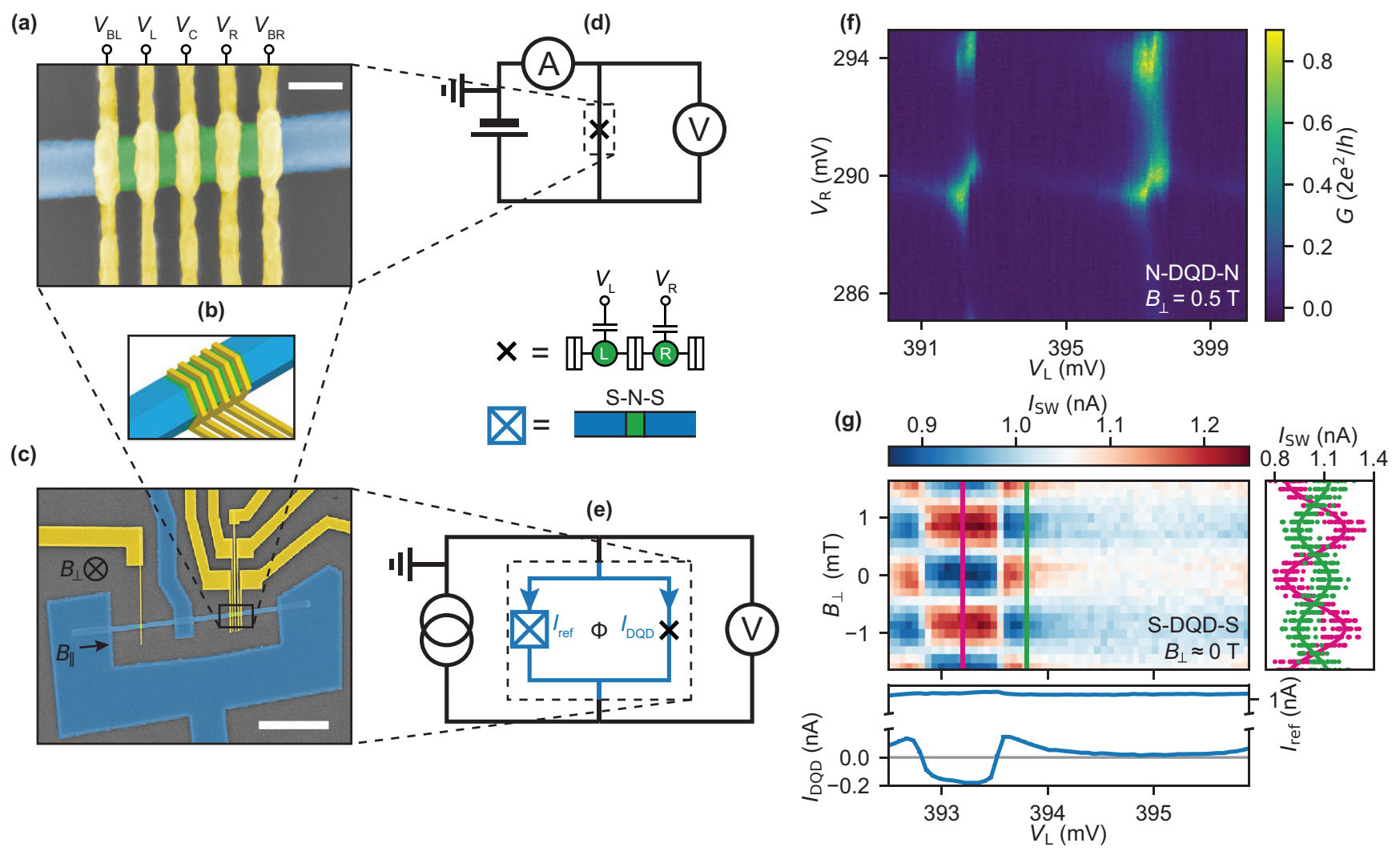

FIG. 1. Device layout and characterization. (a) Color-enhanced electron micrograph of the nanowire DQD junction with five wrap-around gates (yellow) which provide the confining potential. The $V_{\mathrm{BL}}, V_{\mathrm{C}}$, and $V_{\mathrm{BR}}$ gate voltages define the barriers, while $V_{\mathrm{L}}$ and $V_{\mathrm{R}}$ control the number of electrons on the dots. The aluminum shell (blue) is selectively etched away in the weak link section (green denotes bare InAs). The scale bar denotes $100 \mathrm{~nm}$. (b) Perspective drawing of the DQD junction highlighting the conformal gates. (c) Color-enhanced electron micrograph of the DC SQUID made of sputtered NbTiN film (in blue) with the reference junction in the left arm and the DQD junction in the right arm. The scale bar denotes $2 \mu \mathrm{m}$. (d) The circuit diagram for the normal-state characterization with the reference arm depleted. (e) The measurement scheme of the switching current measurements in the SQUID geometry. (f) Charge stability diagram of the DQD in the normal state at a large magnetic field $B_{\perp}=0.5 \mathrm{~T}$. (g) Switching current color map through three charge states of the DQD and the flux $\Phi$ induced by a small $B_{\perp}$. Each pixel is an average of 18 measurements. The side panel shows all switching current data taken along the magenta and green line, respectively. The solid lines denote the sinusoidal fit yielding the signful oscillation amplitude $I_{\mathrm{DQD}}$ and offset $I_{\text {ref }}$ (see text). The standard deviation of the phase is $6 \times 10^{-3} \pi$ and $1.2 \times 10^{-2} \pi$ for the magenta and green lines, respectively. The bottom panel displays the fitted $I_{\text {ref }}$ and $I_{\mathrm{DQD}}$. The DQD was tuned along the total energy axis [see the solid black line in Fig. 2(a)] and we display the corresponding $V_{\mathrm{L}}$ range on the horizontal axis.

selectively removed where the DQD and the reference arm would be defined. Next, we created the SQUID loop from a sputtered NbTiN superconducting film, and covered the device with a 10 -nm-thick $\mathrm{AlO}_{x}$ dielectric by conformal atomic layer deposition. Finally, 40-nm-wide and 50-nmthick Ti/Au gates [in yellow in Fig. 1(a)] were evaporated under three angles to ensure a conformal coverage around the wire [schematically shown in Fig. 1(b)]. Five gates defined the DQD (on the right) and a single gate controlled the reference arm [on the left in Fig. 1(c)]. Details on the device fabrication are shown in the Supplemental Material [27]. All of our measurements were performed in a dilution refrigerator with a base temperature of approximately $30 \mathrm{mK}$.

We first characterize the DQD with the leads driven to the normal state by a large magnetic field, $B_{\perp}=0.5 \mathrm{~T}$. We measure the differential conductance $d I / d V$ of the DQD with the reference arm fully depleted [Fig. 1(d)]. We control the coupling to the leads with the gate voltages $V_{\mathrm{BL}}$ and $V_{\mathrm{BR}}$, and the interdot coupling is tuned by $V_{\mathrm{C}}$ [Fig. 1(a)]. A character- istic honeycomb diagram is plotted in Fig. 1(f), where the charge occupancy of the dots $\left(n_{\mathrm{L}}, n_{\mathrm{R}}\right)$ is set by the voltages applied on the two plunger gates, $V_{\mathrm{L}}$ and $V_{\mathrm{R}}$.

We perform the CPR measurements with the leads being superconducting and with the reference arm of the SQUID opened with its electrostatic gate so that it exhibits a higher critical current than the DQD arm. Due to this asymmetry, the phase drop over the DQD junction is determined by the magnetic flux $\Phi$ through the SQUID loop area [Fig. 1(e)] [14,28], which is proportional to the applied magnetic field $B_{\perp}$. We measure the switching current $I_{\mathrm{SW}}$ of the SQUID by ramping a current bias in a sawtooth wave form and recording the bias current value when the junction switches to the resistive state marked by a threshold voltage drop of the order of $10 \mu \mathrm{V}$. We show a typical data set in Fig. 1(g), where each pixel in the main panel is an average of 18 measurements. The right side panel shows the raw data points at two plunger gate settings denoted by the magenta and green lines in the main panel, as well as the fitted sinusoidal curves in the following functional 


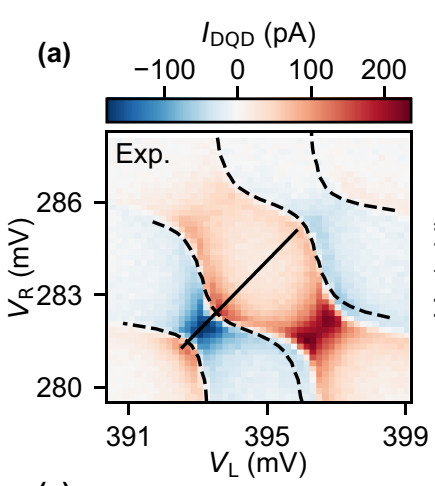

(c)

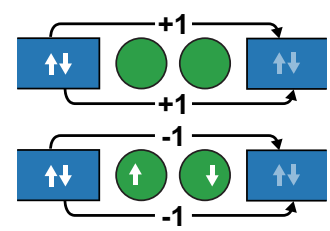

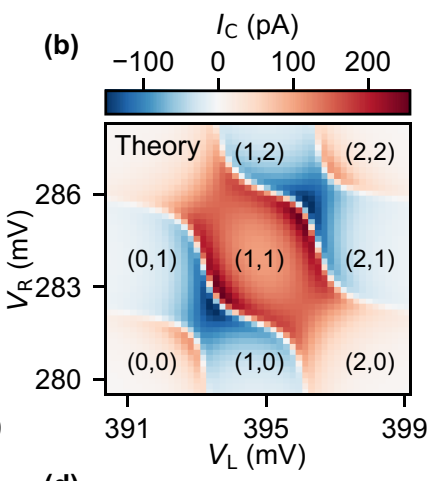

(d)

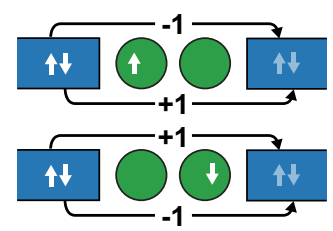

FIG. 2. The supercurrent charge-stability diagram at zero magnetic field. (a) Color map of the measured $I_{\mathrm{DQD}}$ as a function of the plunger gate voltages $V_{\mathrm{L}}$ and $V_{\mathrm{R}}$ revealing a supercurrent sign reversal between the adjacent total charge sectors. The dashed lines denote the numerically calculated charge boundaries (see the text). Measurements along the solid line are shown in Figs. 1(g) and 3(a). (b) The ZBW calculation of the critical current $I_{\mathrm{C}}$ of the DQD using the same parameters. The charge occupation of the dots is indicated in parentheses. Visual representations of a Cooper pair transfer when the DQD has an (c) even and (d) odd charge occupation. The \pm 1 values indicate the spin permutation parity for each spin species, which yields a supercurrent reversal for an odd charge occupation of the DQD (see the text).

form,

$$
I_{\mathrm{SW}}=I_{\mathrm{ref}}+I_{\mathrm{DQD}} \sin \varphi,
$$

where $\varphi=2 \pi\left(B_{\perp}-B_{\mathrm{o}}\right) / B_{\mathrm{p}}$, with $B_{\mathrm{p}} \approx 1.7 \mathrm{mT}$ being the magnetic field periodicity corresponding to a flux change equal to the superconducting flux quantum $\Phi_{0}=h / 2 e$ and $B_{0}$ being the offset perpendicular magnetic field. The switching current values $I_{\text {ref }}$ and $I_{\mathrm{DQD}}$ represent the reference arm and the DQD junction contributions, respectively. We show these fitted values as a function of the gate voltage $V_{\mathrm{L}}$ in the lower subpanel of Fig. 1(g), which displays the sign change of $I_{\mathrm{DQD}}$ at the charge state boundaries. We note that the change in the environmental impedance [29] causes a slight modulation of $I_{\text {ref }}$ as well, despite the lack of any capacitive coupling between the two weak links. However, in our measurements $I_{\text {ref }}>5\left|I_{\mathrm{DQD}}\right|$ is always fulfilled, enabling a reliable observation of the supercurrent reversal in the DQD.

In Fig. 2(a), we plot $I_{\mathrm{DQD}}$ as a function of the plunger gate voltages $V_{\mathrm{L}}$ and $V_{\mathrm{R}}$, resulting in the zero magnetic field chargestability diagram of the DQD mapped by the supercurrent. Remarkably, our phase-sensitive measurement directly shows that the supercurrent reversal is associated with the change in the total charge number, and it is absent in the case of internal charge transfers with $\left(n_{\mathrm{L}}, n_{\mathrm{R}}\right) \rightarrow\left(n_{\mathrm{L}} \pm 1, n_{\mathrm{R}} \mp 1\right)$. However, $\left|I_{\mathrm{DQD}}\right|$ exhibits maxima near all charge boundaries, consistently with earlier experiments [25].

We understand these data using a two-orbital Anderson model, where each dot with an on-site charging energy $U_{i}$ hosts a single spinful level at $\varepsilon_{i}$ with the dot index $i=\mathrm{L}, \mathrm{R}$. In the experiment, this corresponds to a quantum dot orbital level spacing which is larger than the charging energy [14]. We consider an interdot charging energy term $U_{\mathrm{C}} n_{\mathrm{L}} n_{\mathrm{R}}$ and an effective interdot tunneling amplitude $t_{\mathrm{C}}$. The tunnel coupling energies to the superconducting leads are denoted by $\Gamma_{\mathrm{L}, \mathrm{R}}$.

We consider the leading term of the supercurrent in the weak-coupling limit where $t_{\mathrm{C}}, \Gamma_{\mathrm{L}}, \Gamma_{\mathrm{R}} \ll \Delta \ll U_{i} \quad[18,30]$, and evaluate the current operator $I(\varphi)=i \frac{e}{\hbar}\left[H, n_{\mathrm{R}}\right]$, where $H$ is the Hamiltonian of the system at a phase difference of $\varphi$ between the superconducting leads (see the Supplemental Material [27]). We numerically evaluate $\langle I(\varphi)\rangle=I_{\mathrm{C}} \sin \varphi$ to find the signful $I_{C}$. We perform a global fit of the calculated sign reversal contours [see the dashed lines in Fig. 2(a)] against the experimental data set and recover $U_{\mathrm{L}}=596.6 \mu \mathrm{eV}, U_{\mathrm{R}}=$ $465.9 \mu \mathrm{eV}, U_{\mathrm{C}}=41.5 \mu \mathrm{eV}$, and $t_{\mathrm{C}}=85 \mu \mathrm{eV}$. We match the critical current amplitude scale with the experimental data by setting $\Gamma_{\mathrm{L}}=\Gamma_{\mathrm{R}}=33.2 \mu \mathrm{eV}$. The width of the even-odd transitions establishes an upper bound on the electron temperature of the DQD, $T<80 \mathrm{mK}$. We use these parameters to display $I_{\mathrm{C}}\left(V_{\mathrm{L}}, V_{\mathrm{R}}\right)$ in Fig. 2(b) and find a good correspondence with the experimental data using a zero bandwidth (ZBW) approximation [25,31] (see the Supplemental Material [27]).

The observed supercurrent reversal [14,32] is linked to the number of permutations of fermion operators required to transfer a spin-singlet Cooper pair through the DQD (see the Supplemental Material [27]). In the weak-coupling limit, this amounts to counting the number of same-spin dot electrons, which each electron in the Cooper pair crosses. Each such crossing contributes with a factor of -1 to $I_{C}$, which we illustrate for a DQD with even [Fig. 2(c)] and odd charge occupations [Fig. 2(d)]. Consequently, the sign of $I_{C}$ is determined by the ground-state charge parity of the DQD.

Next, we focus on the magnetic field dependence of $I_{\mathrm{DQD}}$ [Fig. 3(a)] along the total energy axis [solid line in Fig. 2(a)] spanning both even and odd charge states. At $B_{\|}=0$, a finite $t_{\mathrm{C}}$ results in a singlet-triplet splitting $\Delta_{\mathrm{ST}}$ in the even occupied $(1,1)$ charge state [1]. We model the DQD with an effective identical $g$-factor on both dots, which results in a spin-polarized triplet ground state above a threshold magnetic field, $B_{\mathrm{ST}}=\Delta_{\mathrm{ST}} /\left(g^{*} \mu_{\mathrm{B}}\right)$. To account for spin-orbit coupling, we refine our interdot tunneling Hamiltonian to include both spin-conserving and spin-flip tunneling amplitudes $t_{0}$ and $t_{\mathrm{x}}$, resulting in an effective $t_{\mathrm{C}}=\sqrt{t_{0}^{2}+t_{\mathrm{x}}^{2}}$ (see the Supplemental Material [27]).

With a global fit to the experimental data [Figs. 3(a) and $3(\mathrm{~b})]$, we extract $t_{0}=80 \mu \mathrm{eV}, t_{\mathrm{x}}=30 \mu \mathrm{eV}$, and $g^{*}=15.9$. This $g$-factor is in agreement with earlier experimental values measured on InAs quantum dots [5,33-35] and ballistic channels with superconducting leads $[26,36]$. We estimate the spin-orbit length $l_{\mathrm{SO}}=l_{\text {dot }} t_{0} /\left(\sqrt{2} t_{\mathrm{x}}\right) \approx 75 \mathrm{~nm}$ [37], using the gate pitch as an estimate of the dot length, $l_{\mathrm{dot}}=40 \mathrm{~nm}$. This coupling length yields an energy scale $E_{\mathrm{SO}}=\hbar^{2} /\left(2 m^{*} l_{\mathrm{SO}}^{2}\right)=$ $290 \mu \mathrm{eV}$ with an effective electron mass of $m^{*}=0.023 m_{e}$, which is similar to earlier experimental results on semiconductor nanowires in the presence of strong electrostatic confinement [38,39]. 

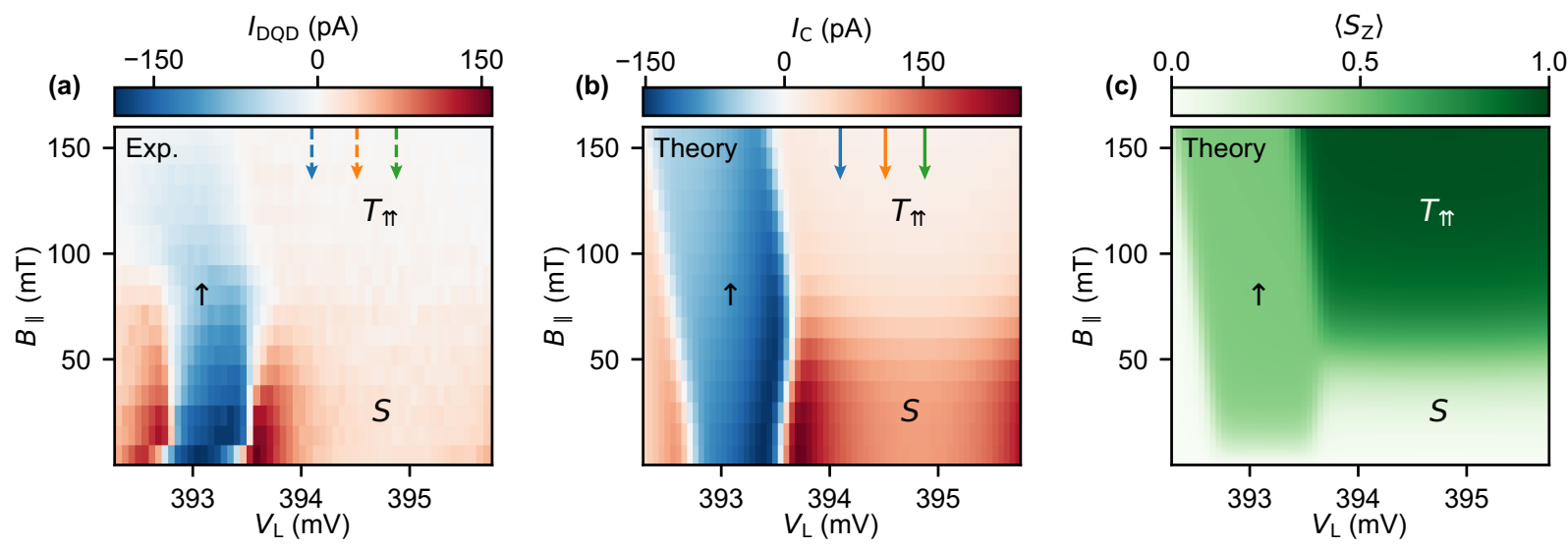

FIG. 3. The superconducting DQD in finite magnetic fields. (a) The measured signful supercurrent oscillation amplitude $I_{\mathrm{DQD}}$ as a function of the total energy [see the solid line in Fig. 2(a)] and magnetic field. Note the slight charge shift between the zero magnetic field line and the rest of the data. (b) The corresponding ZBW calculation of the signful critical current (see the text). (c) The calculated spin expectation value in the ground state showing the singlet to triplet transition in the even occupied state as a function of the magnetic field. In (b) and (c), we use the parameters extracted in Fig. 2(b).

In Fig. 3(c), we plot the calculated expectation value $\left\langle S_{Z}\right\rangle$ of the total spin $z$ component of the DQD, which visualizes the transition between the spin-singlet state $\left\langle S_{Z}\right\rangle=0$ and the spin-polarized triplet state, where $\left\langle S_{Z}\right\rangle=1$, as a function of the magnetic field. This transition point at $B_{\mathrm{ST}}$ is accompanied by a drop of the critical current in the $(1,1)$ sector, however, this sudden decrease is absent in the odd sector [see the blue regions in Fig. 3(b)]. We note that the gradual global decrease in $I_{\mathrm{DQD}}$ is consistent with the orbital effect of the magnetic field applied along the nanowire [40].

We analyze these data in Fig. 4, where we first find the charge state boundary at each value of $B_{\|}$at $I_{\mathrm{DQD}}=0$ [blue dots and error bars in Fig. 4(a)] and overlay the calculated boundary [black solid line, corresponding to Fig. 3(b)]. We quantify $B_{\mathrm{ST}} \approx 80 \mathrm{mT}$, which agrees consistently with the characteristic cutoff magnetic field of $I_{\mathrm{DQD}}$ at several plunger gate values [dots in Fig. 4(b), colors corresponding to the arrows in Fig. 3(a)]. However, we observe a deviation between the calculated and measured charge boundary near $B_{\mathrm{ST}}$, which may stem from the microscopic details of the spin-orbit coupling that our model does not account for. We find an excellent agreement with the calculated critical current $I_{\mathrm{C}}(B)$ [solid lines in Fig. 4(b)] with a common scaling factor of 0.29 , which may be the result of the reduced switching current inside the charge state due to thermal activation compared to the corresponding critical current [29].

The suppression of the Josephson supercurrent through a DQD in the spin-triplet sector can be understood considering the virtual states involved in the Cooper pair transfer. Starting from the $(1,1) T_{\Uparrow}$ state close to the charge boundary with the single occupation sector, we always encounter a virtual state with a double occupation on one of the dots [magenta circle in Fig. 4(c)]. In the $U \gg \Delta$ limit corresponding to our experiments, this configuration is energetically unfavorable and suppresses Cooper pair tunneling. In contrast, a spin-singlet starting condition can avoid this configuration [Fig. 4(d)]. We finally note that the opposite limit, where $U \ll \Delta$, also leads to a triplet supercurrent blockade [20] (see the Supplemental
Material [27]), which persists with a finite residual supercurrent in the spin-triplet state when $U \sim \Delta$.

In conclusion, we directly measured the supercurrent reversal associated with the even-odd charge occupation in an InAs DQD, where the large level spacing allows us to use a single orbital for each dot in our quantitative modeling. In the $(1,1)$ charge sector, we showed that the singlet to triplet transition is accompanied by a supercurrent blockade. This enables a direct spin to supercurrent conversion [36,41] in
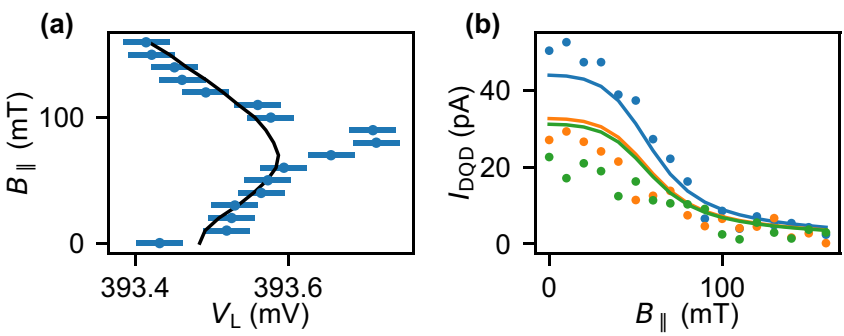

(c)

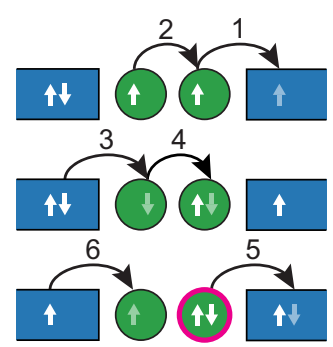

(d)

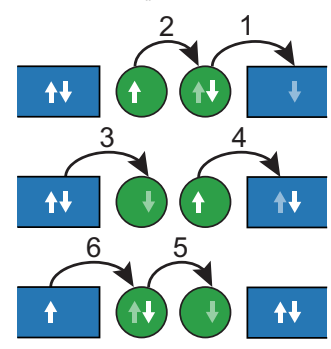

FIG. 4. Triplet-blockaded supercurrent. (a) The measured (blue dots and error bars) and calculated (black solid line) even-odd charge boundary extracted from Figs. 3(a) and 3(b). (b) Dots: The measured $I_{\mathrm{DQD}}$ at three plunger gate settings in the even $(1,1)$ sector [see the corresponding arrows in Fig. 3(a)]. Scaled theoretical values are shown as solid lines (see the text). Representative sixth-order tunneling processes are shown (c) in the $T_{\uparrow}$ and (d) in the singlet regime. The white arrows denote an initial occupied electron state including the spin. The gray arrows visualize the final state for each numbered process. 
hybrid semiconductor nanodevices [30] used for quantum information processing.

Raw data sets and computer code are available at the Zenodo repository [42].

The authors thank J. Danon for helpful discussions, A. Proutski and D. Laroche for their technical input, as well as J. Mensingh, M. Sarsby, O. Benningshof, and R. N. Schouten for technical assistance. This work was supported by the QuantERA project SuperTop, by the NANOCOHYBRI
COST Action No. CA16218, by the Netherlands Organization for Scientific Research (NWO), by the Danish National Research Foundation, by the National Research Development and Innovation Office of Hungary within the Quantum Technology National Excellence Program (Project No. 20171.2.1-NKP-2017-00001), under OTKA Grants No. 124723 and No. 132146, and the BME Nanotechnology and Materials Science TKP2020 IE grant (BME IE-NAT TKP2020), and by the European Union's Horizon 2020 research and innovation programme under Grants No. 716655 (ERC Stg HEMs-DAM), No. 804988 (ERC Stg SiMS), and No. 828948 (FET Open AndQC).
[1] R. Hanson, L. P. Kouwenhoven, J. R. Petta, S. Tarucha, and L. M. K. Vandersypen, Spins in few-electron quantum dots, Rev. Mod. Phys. 79, 1217 (2007).

[2] D. Loss and D. P. DiVincenzo, Quantum computation with quantum dots, Phys. Rev. A 57, 120 (1998).

[3] W. G. Van der Wiel, S. De Franceschi, J. M. Elzerman, T. Fujisawa, S. Tarucha, and L. P. Kouwenhoven, Electron transport through double quantum dots, Rev. Mod. Phys. 75, 1 (2002).

[4] K. C. Nowack, F. H. L. Koppens, Y. V. Nazarov, and L. M. K. Vandersypen, Coherent control of a single electron spin with electric fields, Science 318, 1430 (2007).

[5] S. Nadj-Perge, S. M. Frolov, E. P. A. M. Bakkers, and L. P. Kouwenhoven, Spin-orbit qubit in a semiconductor nanowire, Nature (London) 468, 1084 (2010).

[6] M. Field, C. G. Smith, M. Pepper, D. A. Ritchie, J. E. F. Frost, G. A. C. Jones, and D. G. Hasko, Measurements of Coulomb Blockade with a Noninvasive Voltage Probe, Phys. Rev. Lett. 70, 1311 (1993).

[7] T. Frey, P. J. Leek, M. Beck, A. Blais, T. Ihn, K. Ensslin, and A. Wallraff, Dipole Coupling of a Double Quantum Dot to a Microwave Resonator, Phys. Rev. Lett. 108, 046807 (2012).

[8] K. D. Petersson, L. W. McFaul, M. D. Schroer, M. Jung, J. M. Taylor, A. A. Houck, and J. R. Petta, Circuit quantum electrodynamics with a spin qubit, Nature (London) 490, 380 (2012).

[9] B. D. Josephson, Possible new effects in superconductive tunnelling, Phys. Lett. 1, 251 (1962).

[10] A. A. Golubov, M. Y. Kupriyanov, and E. Il'ichev, The currentphase relation in Josephson junctions, Rev. Mod. Phys. 76, 411 (2004).

[11] V. V. Ryazanov, V. A. Oboznov, A. Y. Rusanov, A. V. Veretennikov, A. A. Golubov, and J. Aarts, Coupling of Two Superconductors through a Ferromagnet: Evidence for a $\pi$ Junction, Phys. Rev. Lett. 86, 2427 (2001).

[12] T. Kontos, M. Aprili, J. Lesueur, F. Genêt, B. Stephanidis, and R. Boursier, Josephson Junction through a Thin Ferromagnetic Layer: Negative Coupling, Phys. Rev. Lett. 89, 137007 (2002).

[13] J. J. A. Baselmans, A. F. Morpurgo, B. J. van Wees, and T. M. Klapwijk, Reversing the direction of the supercurrent in a controllable Josephson junction, Nature (London) 397, 43 (1999).

[14] J. A. van Dam, Y. V. Nazarov, E. P. A. M. Bakkers, S. De Franceschi, and L. P. Kouwenhoven, Supercurrent reversal in quantum dots, Nature (London) 442, 667 (2006).

[15] R. Delagrange, R. Weil, A. Kasumov, M. Ferrier, H. Bouchiat, and R. Deblock, $0-\pi$ quantum transition in a carbon nanotube
Josephson junction: Universal phase dependence and orbital degeneracy, Phys. Rev. B 93, 195437 (2016).

[16] M.-S. Choi, C. Bruder, and D. Loss, Spin-dependent Josephson current through double quantum dots and measurement of entangled electron states, Phys. Rev. B 62, 13569 (2000).

[17] Y. Zhu, Q.-F. Sun, and T.-H. Lin, Probing spin states of coupled quantum dots by a dc Josephson current, Phys. Rev. B 66, 085306 (2002).

[18] A. Martín-Rodero and A. L. Yeyati, Josephson and Andreev transport through quantum dots, Adv. Phys. 60, 899 (2011).

[19] C. Karrasch, S. Andergassen, and V. Meden, Supercurrent through a multilevel quantum dot close to singlet-triplet degeneracy, Phys. Rev. B 84, 134512 (2011).

[20] S. Droste, S. Andergassen, and J. Splettstoesser, Josephson current through interacting double quantum dots with spin-orbit coupling, J. Phys.: Condens. Matter 24, 415301 (2012).

[21] A. Brunetti, A. Zazunov, A. Kundu, and R. Egger, Anomalous Josephson current, incipient time-reversal symmetry breaking, and Majorana bound states in interacting multilevel dots, Phys. Rev. B 88, 144515 (2013).

[22] V. Pokorný, M. Žonda, G. Loukeris, and T. Novotný, Second order perturbation theory for a superconducting double quantum dot, JPS Conf. Proc. 30, 011002 (2020).

[23] P. Krogstrup, N. Ziino, W. Chang, S. Albrecht, M. Madsen, E. Johnson, J. Nygård, C. Marcus, and T. Jespersen, Epitaxy of semiconductor-superconductor nanowires, Nat. Mater. 14, 400 (2015).

[24] D. Szombati, S. Nadj-Perge, D. Car, S. Plissard, E. Bakkers, and L. Kouwenhoven, Josephson $\phi_{0}$-junction in nanowire quantum dots, Nat. Phys. 12, 568 (2016).

[25] J. C. Estrada Saldaña, A. Vekris, G. Steffensen, R. Žitko, P. Krogstrup, J. Paaske, K. Grove-Rasmussen, and J. Nygård, Supercurrent in a Double Quantum Dot, Phys. Rev. Lett. 121, 257701 (2018).

[26] D. J. van Woerkom, A. Proutski, B. Van Heck, D. Bouman, J. I. Väyrynen, L. I. Glazman, P. Krogstrup, J. Nygård, L. P. Kouwenhoven, and A. Geresdi, Microwave spectroscopy of spinful Andreev bound states in ballistic semiconductor Josephson junctions, Nat. Phys. 13, 876 (2017).

[27] See Supplemental Material at http://link.aps.org/supplemental/ 10.1103/PhysRevB.102.220505 for the details of sample fabrication, device paramaters, and numerical modeling.

[28] M. L. Della Rocca, M. Chauvin, B. Huard, H. Pothier, D. Esteve, and C. Urbina, Measurement of the Current-Phase 
Relation of Superconducting Atomic Contacts, Phys. Rev. Lett. 99, 127005 (2007).

[29] Y. Ivanchenko and L. Zil'Berman, The Josephson effect in small tunnel contacts, Zh. Eksp. Teor. Fiz. 55, 2395 (1968) [Sov. Phys. JETP 28, 1272 (1969)].

[30] S. De Franceschi, L. Kouwenhoven, C. Schonenberger, and W. Wernsdorfer, Hybrid superconductor-quantum dot devices, Nat. Nanotechnol. 5, 703 (2010).

[31] E. Vecino, A. Martín-Rodero, and A. Levy Yeyati, Josephson current through a correlated quantum level: Andreev states and $\pi$ junction behavior, Phys. Rev. B 68, 035105 (2003).

[32] B. I. Spivak and S. A. Kivelson, Negative local superfluid densities: The difference between dirty superconductors and dirty Bose liquids, Phys. Rev. B 43, 3740(R) (1991).

[33] C. Fasth, A. Fuhrer, L. Samuelson, V. N. Golovach, and D. Loss, Direct Measurement of the Spin-Orbit Interaction in a Two-Electron InAs Nanowire Quantum Dot, Phys. Rev. Lett. 98, 266801 (2007).

[34] S. Csonka, L. Hofstetter, F. Freitag, S. Oberholzer, C. Schonenberger, T. S. Jespersen, M. Aagesen, and J. Nygård, Giant fluctuations and gate control of the $g$-factor in InAs nanowire quantum dots, Nano Lett. 8, 3932 (2008).

[35] M. D. Schroer, K. D. Petersson, M. Jung, and J. R. Petta, Field Tuning the $g$ Factor in InAs Nanowire Double Quantum Dots, Phys. Rev. Lett. 107, 176811 (2011).

[36] L. Tosi, C. Metzger, M. F. Goffman, C. Urbina, H. Pothier, S. Park, A. L. Yeyati, J. Nygård, and P. Krogstrup, Spin-Orbit
Splitting of Andreev States Revealed by Microwave Spectroscopy, Phys. Rev. X 9, 011010 (2019).

[37] J. Danon, Spin-flip phonon-mediated charge relaxation in double quantum dots, Phys. Rev. B 88, 075306 (2013).

[38] S. Nadj-Perge, V. S. Pribiag, J. W. G. van den Berg, K. Zuo, S. R. Plissard, E. P. A. M. Bakkers, S. M. Frolov, and L. P. Kouwenhoven, Spectroscopy of Spin-Orbit Quantum Bits in Indium Antimonide Nanowires, Phys. Rev. Lett. 108, 166801 (2012).

[39] Z. Scherübl, G. Fülöp, M. H. Madsen, J. Nygård, and S. Csonka, Electrical tuning of Rashba spin-orbit interaction in multigated InAs nanowires, Phys. Rev. B 94, 035444 (2016).

[40] K. Zuo, V. Mourik, D. B. Szombati, B. Nijholt, D. J. van Woerkom, A. Geresdi, J. Chen, V. P. Ostroukh, A. R. Akhmerov, S. R. Plissard, D. Car, E. P. A. M. Bakkers, D. I. Pikulin, L. P. Kouwenhoven, and S. M. Frolov, Supercurrent Interference in Few-Mode Nanowire Josephson Junctions, Phys. Rev. Lett. 119, 187704 (2017).

[41] M. Hays, V. Fatemi, K. Serniak, D. Bouman, S. Diamond, G. de Lange, P. Krogstrup, J. Nygård, A. Geresdi, and M. H. Devoret, Continuous monitoring of a trapped superconducting spin, Nat. Phys. 16, 1103 (2020).

[42] D. Bouman, R. J. J. van Gulik, G. Steffensen, D. Pataki, P. Boross, P. Krogstrup, J. Nygård, J. Paaske, A. Pályi, and A. Geresdi, Triplet-blockaded Josephson supercurrent in double quantum dots (2020), http://doi.org/10.5281/zenodo.3978236. 